\title{
BMJ Open Exposure to outdoor air pollution and its human-related health outcomes: an evidence gap map
}

\author{
Zhuanlan Sun (D) , ${ }^{1}$ Demi Zhu ${ }^{2}$
}

To cite: Sun Z, Zhu D. Exposure to outdoor air pollution and its humanrelated health outcomes: an evidence gap map. BMJ Open 2019;9:e031312. doi:10.1136/ bmjopen-2019-031312

- Prepublication history and additional material for this paper are available online. To view these files, please visit the journal online (http://dx.doi. org/10.1136/bmjopen-2019031312).

Received 01 May 2019 Accepted 18 November 2019

Check for updates

(c) Author(s) (or their employer(s)) 2019. Re-use permitted under CC BY-NC. No commercial re-use. See rights and permissions. Published by BMJ.

${ }^{1}$ Department of Management Science and Engineering, Tongji University, Shanghai, China ${ }^{2}$ Department of Comparative Politics, Shanghai Jiaotong University, Shanghai, China

Correspondence to

Professor Demi Zhu;

Zhudemi@sjtu.edu.cn

\section{ABSTRACT}

Objectives Outdoor air pollution is a serious environmental problem worldwide. Current systematic reviews (SRs) and meta-analyses (MAs) mostly focused on some specific health outcomes or some specific air pollution.

Design This evidence gap map (EGM) is to identify existing gaps from SRs and MAs and report them in broad topic areas.

Data sources PubMed, Cochrane, Scopus and Web of Science were searched from their inception until June 2018. Citations and reference lists were traced.

Eligibility criteria SRs and MAs that investigated the impact of outdoor air pollution on human health outcomes were collected. This study excluded original articles and qualitative review articles.

Data extraction and synthesis Characteristics of the included SRs and MAs were extracted and summarised. Extracted data included authors, publication year, location of the corresponding author(s), publication journal discipline, study design, study duration, sample size, study region, target population, types of air pollution and health outcomes.

Results Asia and North America published $93 \%$ of SRs and MAs included in this EGM. $31 \%$ of the SRs and MAs (27/86) included primary studies conducted in 5-10 countries. Their publication trends have increased during the last 10 years. A total of 2864 primary studies was included. The median number of included primary studies was 20 (range, 7-167). Cohort studies, case cross-over studies and time-series studies were the top three most used study designs. The mostly researched population was the group of all ages (46/86, 53\%). Cardiovascular diseases, respiratory diseases and health service records were mostly reported. A lack of definite diagnostic criteria, unclear reporting of air pollution exposure and time period of primary studies were the main research gaps. Conclusions This EGM provided a visual overview of health outcomes affected by outdoor air pollution exposure. Future research should focus on chronic diseases, cancer and mental disorders.

\section{INTRODUCTION}

Outdoor air pollution is a serious environmental problem and a major public health concern worldwide; it has caused approximately 4.2 million global deaths in 2016 . $^{1}$ It is a severe invisible killer posing serious

\section{Strengths and limitations of this study}

This study established an evidence gap map (EGM), as a two-dimensional matrix, of outdoor air pollution and health outcomes; the rows and columns of the array included all categories of outdoor air pollution and human health-related outcomes, respectively.

- This EGM focused on a broad range of air pollution and human health-related outcomes based on a systematic search of four databases from their inception until June 2018.

- The bubble plot was used to visualise the evidence gap, the colour represented the confidence of included systematic reviews (SRs) and the size indicated the number of included SRs.

- Only SRs and meta-analyses (MAs) were included in this EGM.

- The publication language of included SRs and MAs was restricted to English, thus possibly missing pertinent information written in other languages.

human health hazards, especially in developing countries, speeded up by industrialisation and urbanisation. ${ }^{2}$ There are many common types of air pollutants, such as fine particulate matter $\left(\mathrm{PM}_{2.5}\right.$ and $\left.\mathrm{PM}_{10}\right)$, ozone $\left(\mathrm{O}_{3}\right)$, carbon monoxide $(\mathrm{CO})$, sulfur dioxide $\left(\mathrm{SO}_{2}\right)$, nitrogen oxides $\left(\mathrm{NO}_{2}\right.$ and $\left.\mathrm{NO}_{\mathrm{x}}\right)$, volatile organic pollutants and some other toxic air pollutants. The human health-related outcomes affected by such air pollutants have been studied for decades. Researchers from various disciplines all over the world have shown constant interest in the health effects of outdoor air pollution, such as cardiovascular disease, respiratory disease, cancer, Alzheimer's disease and so on. ${ }^{3-6}$

Systematic reviews (SRs) aim to synthesis all relevant high-quality evidence on a specific topic in terms of the health outcomes of outdoor air pollution. ${ }^{7}$ It was reported that outdoor $\mathrm{NO}_{2}$ exposure triggered asthma, ${ }^{89}$ other studies identified human mortality was positively associated with $\mathrm{PM}_{2.5}$ exposure. ${ }^{1011}$ However, many studies have focused on interactive effects with regard to several types of 
health outcomes affected by various air pollutants, ${ }^{12} 13$ and there are some overlapping SRs implying inconsistent results. ${ }^{1415}$ The specific topic of SR findings on particular factors cannot be used to provide a comprehensive overview of health outcomes related to air pollution.

To overcome this barrier, some novel knowledge synthesis approaches (eg, scoping review, systematic mapping, evidence mapping, etc) have been developed to evaluate the overall effects of evidence on a broader area, highlighting both current knowledge and gaps in evidence. ${ }^{16-19}$ Evidence gap map (EGM), an emerging evidence synthesis method, was proposed to systematically and comprehensively assess intervention effects and outcomes in a user-friendly two-dimensional matrix framework. It can also efficiently visualise the existing evidence in a map with a critical quality appraisal for policy and research implications. ${ }^{2021}$

EGM can inform a strategic approach to build evidence based on particular issues. Water, sanitation and hygiene interventions were evaluated by the International Initiative for Impact Evaluation (3ie) to inform policy decisions and address the evidence gap. ${ }^{21}$ The 3ie is a global collaboration focus on evidence synthesis. To our knowledge, there is little research that has overviewed health outcomes, related to outdoor air pollution exposure, using EGM.

We constructed an EGM on the health effects of outdoor air pollution by comprehensively summarising existing SRs and meta-analyses (MAs). The main purpose of this EGM was to identify the evidence gaps for potential research prioritisation. In addition, it also can be used for evidence-based environmental policymaking support.

\section{MATERIALS AND METHODS}

EGM is a newly developed method of knowledge synthesis, which is similar to evidence mapping. ${ }^{18}$ There is no standard methodology guideline available for evidence mapping currently. ${ }^{22}$ We followed the methodology framework presented by Snilstveit et al to conduct this EGM. ${ }^{21}$ The establishment of the framework was divided into six steps: developing scope; setting inclusive criteria; searching relevant studies; selecting studies; extracting data and appraising quality; summarising results.

\section{Scope development}

The established framework was a two-dimensional matrix of outdoor air pollution and health outcomes. It was derived from the major policies of interest and existing academic literature. The rows and columns of the array included all categories of outdoor air pollution and human health-related outcomes, respectively.

To improve the quality of EGM, stakeholders, including academic researchers, policymakers, practitioners and funders, were recruited. The academic researchers are from the fields of environmental science, public health, epidemiology and economics. Some researchers have interdisciplinary research experience. The policymakers, funders and practitioners are from environmental protection administrations and public health administrations. A consultation with key stakeholders was conducted to ensure the acceptance of the research framework. It was carried out by a process of study inclusion, literature search and data extraction. All the key stakeholders were invited to take part in the consultation to determine the scope and the technologies of the EGM.

\section{Study inclusion criteria}

Following 3ie guidelines, we included SR and MA studies, and summarised all the available evidence on human health-related outcomes affected by particular air pollution. ${ }^{23}$

\section{Study search}

Four electronic databases, PubMed, Cochrane, Scopus and Web of Science, were searched from their inception until June 2018. The language was restricted to English. We compiled a list of outdoor air pollution and health outcome terms by reviewing the potential text words in the titles or abstracts of the most pertinent literature (online supplementary table $\mathrm{S} 1$ ); this process was conducted by consulting with experts who are members of the Committee on Public Health and Urban Environment Management in China. We then used advanced database search strategies on key words, combined with Boolean operators (AND, OR, NOT) and wild card symbols $(*)$, so as to search for the potentially relevant literature (online supplementary table S2). This study focused on the health outcomes affected by outdoor air pollution, thus, the term 'air pollution' which contains some contaminants inside private and public buildings was not included in the search strategy. To avoid missing any relevant literature, we subsequently traced citations and reference lists of literature to generate more relevant SR and MA studies.

\section{Inclusion assessment}

The first step of inclusion assessment involved screening the titles and abstracts of SRs and MAs by two reviewers independently. The inclusion and exclusion criteria for EGM were discussed by the team members at the

Table 1 Inclusion and exclusion criteria used in this EGM for eligible studies

\begin{tabular}{lc}
\hline Inclusion criteria & Exclusion criteria \\
\hline Study types & Study type \\
\hline SRs and MAs & Indoor air pollution \\
\hline Study methods & Primary studies \\
\multicolumn{1}{c}{ Quantitative SRs } & Study methods \\
Study conclusion & Qualitative reviews only \\
$\begin{array}{l}\text { Data supported outcomes } \\
\text { relevant to outdoor air pollution }\end{array}$ & \\
\hline
\end{tabular}

EGM, evidence gap map; MAs, meta-analyses; SRs, systematic reviews. 
Table 2 Information to be collected during the data extraction stage

\begin{tabular}{llll}
\hline Study design & Study population & Exposures & Outcome information \\
\hline Author(s) & Sample size & Types of air pollution & Health outcomes \\
Publication Year & Study region & Key results \\
$\begin{array}{l}\text { Location of the corresponding } \\
\text { author(s) }\end{array}$ & Target population & \\
\hline
\end{tabular}

Publication journal discipline ${ }^{\mathrm{a}}$

Study design

Study duration

a: The discipline catalogue is from the Journal Citation Report published by Web of Science.

beginning of this process (table 1). Primary studies, such as observational studies, time-series studies and causal inference studies, were not included. The definition of outdoor air pollution is that exposure takes place outside of the built environment. Both the health outcomes and outdoor air pollution reported in the SRs and MAs are eligible. Reference management software Mendeley was used to systematically remove duplicated literature and assess potential literature that meets the inclusion criteria.
The second step of the study selection stage was the fulltext retrieval of all potentially eligible articles, which was independently screened by the two reviewers as well. If disagreements occurred between them, a third specialist made the final decision.

\section{Data extraction and critical appraisal}

Characteristics of the included SRs and MAs were extracted and summarised by one reviewer and checked
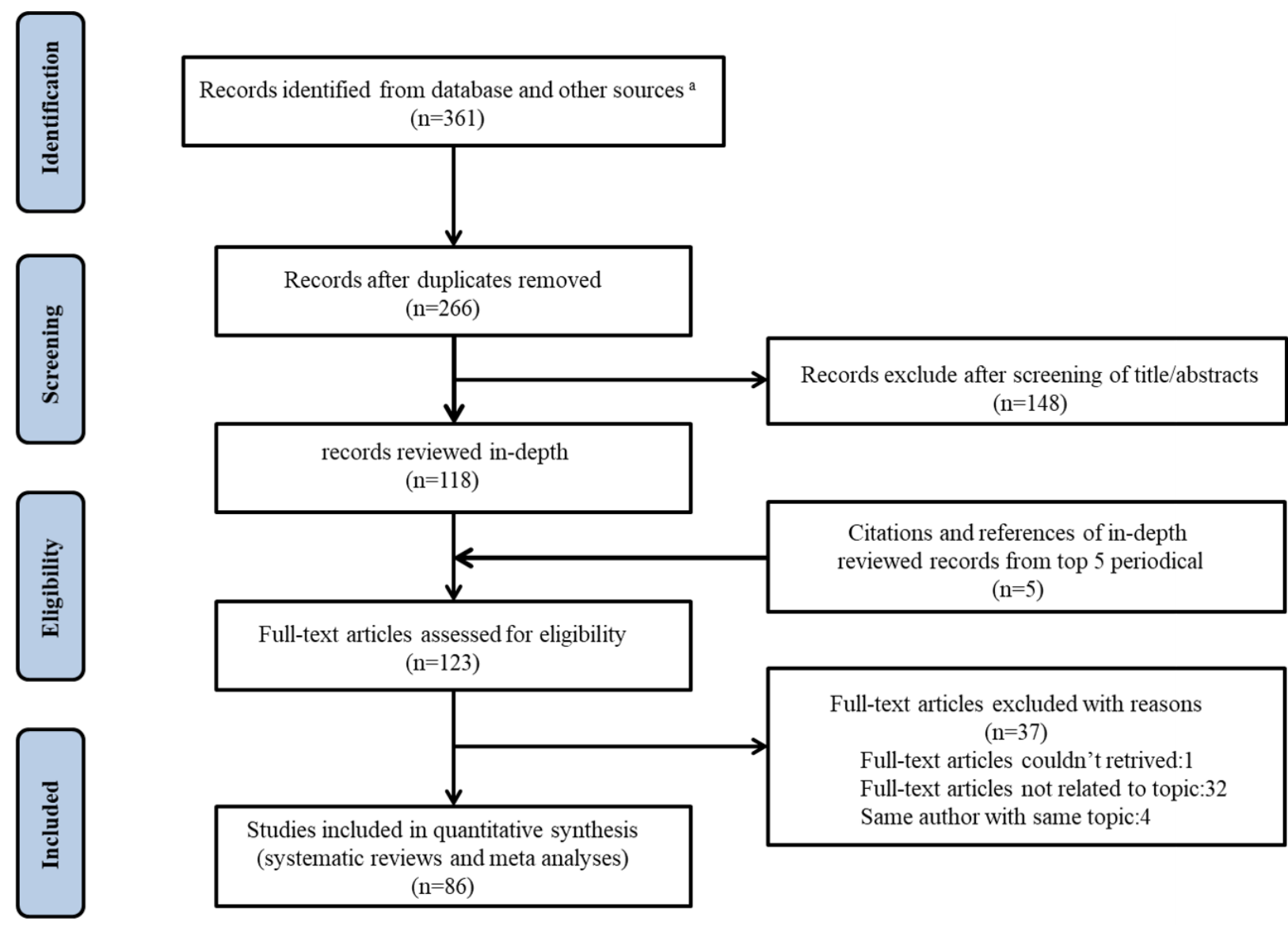

(systematic reviews and meta analyses) $(\mathrm{n}=86)$

a: 59 form PubMed, 216 from Scopus and 86 from Web of Science

Figure 1 Systematic literature search process for eligible SRs and MAs. SR, systematic review; MA, meta-analysis. 
for accuracy by the other. Extracted data included authors, publication year, location of the corresponding author(s), publication journal discipline, study design, study duration, sample size, study region, target population, types of air pollution and health outcomes (table 2). Other categories, such as key results, were extracted based on the advice of some public health researchers and policymakers. We categorised the results of health outcomes into three parts: positive, negative and ambiguous. Positive represents air pollution has harmful effects on human health outcomes. Negative means no harmful effect was found in the MAs. Air pollution was categorised as general air pollution gas, fine particulate matter, other toxic substances and combinations of multiple pollutants. The definition of 'combinations of multiple pollutants' is a mixture of two or more of the following air pollutants: $\mathrm{O}_{3}, \mathrm{SO}_{2}, \mathrm{CO}, \mathrm{NO}_{2}, \mathrm{PM}_{2.5}, \mathrm{PM}_{10}$, suspended particulate matter and total suspended particles.

The potential study designs and the inherent characteristics (strengths and limitations) of each design make the quality assessment complex. ${ }^{24}$ We applied the Supporting the Use for Research Evidence (SURE) checklist to appraise the quality of included SRs and MAs in terms of the confidence, ${ }^{21}$ rating them as high, medium or low confidence. ${ }^{20}$ SURE is a mechanism that aims to strengthen evidence-informed policymaking, which is more appropriate for our research. The SURE checklist was designed to assess three dimensions of the included SRs and MAs: (1) Methods used to identify, include and critically appraise studies. (2) Methods used to analyse the findings. (3) Overall assessment of the reliability of the review. Two authors independently assessed the quality of the included SRs and MAs. Any difference would be resolved by consulting with a third researcher.

\section{Summarisation and visualisation}

To provide a snapshot of what is known and the areas where evidence is lacking, a bubble plot was used to represent the extent of health outcomes affected by outdoor air pollution. We followed the visual representation of 3ie's two-dimensional framework to generate the bubble plot. ${ }^{21}$ In the EGM plot, traffic light colours indicate three levels of confidence of the findings, which were assessed by using the SURE checklist. Red bubbles represent low confidence, yellow ones represent medium and green ones represent high confidence. Bubble size represents the quantity of the corresponding SRs and MAs.

\begin{tabular}{|c|c|c|c|c|c|}
\hline$(n=86)$ & $\mathbf{N}$ & $\%$ & & $\mathbf{N}$ & $\%$ \\
\hline \multicolumn{3}{|l|}{ Continent* } & \multicolumn{3}{|l|}{ Number of original studies } \\
\hline Africa & 1 & 1 & $<10$ studies & 10 & 12 \\
\hline Australia & 2 & 2 & 10 30 studies & 46 & 53 \\
\hline South America & 3 & 3 & 30 60 studies & 13 & 15 \\
\hline North America & 19 & 22 & $>60$ studies & 13 & 15 \\
\hline Asia & 29 & 34 & Not clearly mentioned & 4 & 5 \\
\hline Europe & 32 & 37 & Study period & & \\
\hline \multicolumn{3}{|c|}{ Countries of primary studies included in the SRs and MAs } & $<10$ years & 11 & 13 \\
\hline$<5$ countries & 18 & 21 & 10 20 years & 23 & 27 \\
\hline $5 \sim 10$ countries & 27 & 31 & $>20$ years & 7 & 8 \\
\hline 10 20 countries & 15 & 17 & Not clearly mentioned & 45 & 52 \\
\hline$>20$ countries & 1 & 1 & Types of study designs & & \\
\hline Not clearly mentioned $\dagger$ & 25 & 29 & Single & 13 & 15 \\
\hline Population & & & Multiple & 73 & 85 \\
\hline Infants & 2 & 2 & Study design & & \\
\hline Children and adolescents & 15 & 17 & Cohort & 41 & \\
\hline Adults & 7 & 8 & Case cross-over & 27 & \\
\hline Elderly & 2 & 2 & Time-series & 25 & \\
\hline Women and pregnancy & 4 & 5 & Case-control & 24 & \\
\hline All ages & 46 & 53 & Cross-sectional & 20 & \\
\hline Not specified & 10 & 12 & Sample size (range) & $(83-$ & \\
\hline
\end{tabular}

${ }^{*}$ :The classification of the continent was based on the address of the corresponding authors.

${ }^{\dagger}$ :Not clearlymentioned means no data or information were mentioned in inclusive SRs.

MAs, meta-analyses; SRs, systematic reviews. 


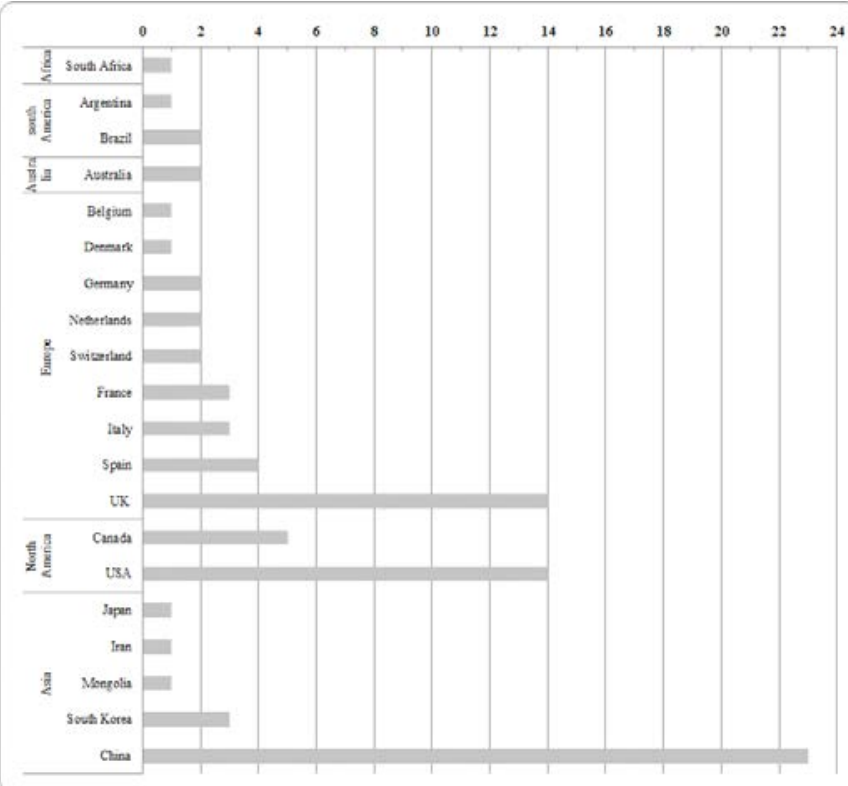

Figure 2 Regionwise and countrywise distributions of eligible SRs and MAs. The data of regions and countries are based on the information of corresponding authors. SRs, systematic reviews; MAs, meta analyses.

\section{Patient and public involvement}

No patients or public were involved in this EGM.

\section{RESULTS}

Figure 1 displays the systematic literature search process for EGM. A literature search of four electronic databases yielded 361 potentially relevant studies. After removing duplicate research, 266 studies were collected. Upon screening the titles and the abstracts, 118 SRs and MAs were assessed as being relevant to the EGM. To avoid missing potential studies, we also manually searched the relevant studies in the top five impact factor periodicals, which were chosen in the collection of our included published SRs and MAs. After tracing the citations and reference lists, five more relevant studies were included at the end of this process. In total, 123 SRs and MAs were included in the full-text screening analysis. Finally, 86 eligible articles were included in our EGM. In all 86 SRs and MAs, a total of 2864 original studies were included. The median number of original studies was 20 (range, 7-167), and the variance value was 31.99 .

\section{Summary of general descriptions of included SRs and MAs}

Most of the 86 included SRs and MAs, were published in Europe, Asia and North America, in a proportion of $37 \%, 34 \%$ and $22 \%$, respectively (table 3 ). As illustrated in figure 2, SRs and MAs published in the top three continents were concentrated in a small group of countries, with China, USA and the UK topping the list. Little evidence came from Africa, Australia and South America, with an overall proportion of only $6 \%$. The distribution of publications was unbalanced.
The population of the included SRs and MAs was categorised into seven subgroups: infants, children and adolescents, adults, women and pregnancy, all ages, elderly and not specified (table 3). The largest population affected by outdoor air pollution fell under the group of all ages $(46 / 86 ; 53 \%)$, followed by the group of children and adolescents under the age of 20 years, with a percentage of $17 \%(15 / 86)$. The infants and elderly groups shared only $2 \%$, however, these two groups of people were mostly affected by air pollution.

Almost half of the available primary studies included in the SRs and MAs were conducted in more than five countries. Of the SRs and MAs, 21\% only included primary studies conducted in less than five countries. The sample size of the primary studies included in the SRs and MAs varied with a wide range, the number of which ranged from less than hundred to millions (range: 83-11 850884 participants).

With regard to study design, $15 \%(13 / 86)$ of the included SRs and MAs were conducted by using a single study design, the 73 remaining SRs and MAs originated from primary studies with multiple study designs. The top five mostly used study designs of the primary studies included in the SRs and MAs were cohort, case crossover, time-series, case-control and cross-sectional, and the corresponding numbers are 41, 27, 25, 24 and 20, respectively. The time period, which means the publication date intervals of primary studies included in the SRs and MAs, were mostly (52\%) unclearly reported.

\section{Publication trends of included SRs and MAs}

The included SRs and MAs were mainly conducted in six research fields. As the cumulative frequency trend chart displays in figure 3 , the number of SRs showed an increasing trend from 2004 to 2018. Most of the included SRs and MAs were published in the last 6 years, with no SRs published before the year 2004. All included SRs and MAs were categorised by using the Journal Citation Report in the Web of Science database. There was an increasing trend in the number of publications in the fields of medicine and public environmental occupational health from 2004 to 2013, and the trend remarkably rose from 2013

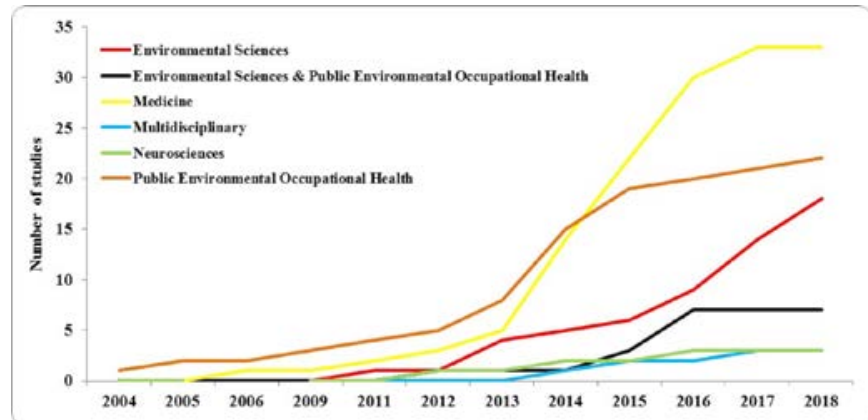

Figure 3 Numbers of inclusive eligible SRs and MAs in six research fields between 2004 and 2018. The number of eligible SRs and MAs saw an increasing trend from 2004 to 2018. Most of the included studies were published in the last 6 years. SRs, systematic reviews; MAs, meta analyses. 


\begin{tabular}{|c|c|}
\hline Health outcome groups & $\begin{array}{l}\text { Health outcome of } \\
\text { inclusive studies }\end{array}$ \\
\hline Respiratory diseases & $\begin{array}{l}\text { Asthma, respiratory } \\
\text { diseases, ALRI }\end{array}$ \\
\hline Chronic diseases & Diabetes \\
\hline Cardiovascular diseases & $\begin{array}{l}\text { Hypertension, BP, COPD, } \\
\text { OHCA, VTE, CVD, } \\
\text { myocardial infarction, } \\
\text { arrhythmia, stroke }\end{array}$ \\
\hline Health service records & $\begin{array}{l}\text { Morbidity, hospital } \\
\text { admissions, ED visits, } \\
\text { mortality }\end{array}$ \\
\hline Cancer & Lung cancer \\
\hline Pregnancy and children & Fertility, pregnancy, birth \\
\hline Mental disorders & $\begin{array}{l}\text { Mental health, cognition, } \\
\text { ASD }\end{array}$ \\
\hline Other diseases & $\begin{array}{l}\text { Physical inactivity, skin } \\
\text { disease, health risks }\end{array}$ \\
\hline
\end{tabular}

ALRI, acute lower respiratory infections; ASD, autism spectrum disorder; BP, blood pressure; COPD, chronic obstructive pulmonary disease; CVD, cardiovascular diseases; ED, emergency department; OHCA, out-of-hospital cardiac arrest; VTE, venous thromboembolism.

till now, especially in medical research. The environmental sciences saw a rising trend since 2011, then the trend spiked up in the years 2012 and 2015, respectively, and reached its peak number in 2017. Three disciplines, including environmental sciences and public environmental occupational health, multidisciplinary and neurosciences, increased smoothly since the year 2011.

\section{Summary by health outcome groups}

We categorised health outcomes into eight groups based on the specialists' suggestions: cardiovascular diseases, chronic diseases, health service records, cancer, mental disorders, respiratory diseases, pregnancy and children and other diseases, which were important features of the EGM visualisation. The categorised list of health outcomes is shown in table 4 .

The inclusive studies in our EGM were summarised based on health outcome groups (table 5). The information about the types of air pollution, the number of primary studies, population groups, sample size and key results were included in table 5. Of the 86 identified SRs and MAs, 24 studies reported cardiovascular diseases as outdoor air pollution related health outcomes, the figures of health service records and respiratory diseases studies that had been reported as an adverse effect of outdoor air pollution exposure were 22 and 19, respectively. Each of the rest five groups of health outcomes accounted for an average SRs and MAs of 4. All SRs and MAs included more than 10 primary studies at least, health outcomes in all ages accounted for the largest groups in all categories, except for those of respiratory diseases category, in which children were the most affected.

Overall, combinations of multiple pollutants were the dominant type of outdoor air pollution, which tended to show a harmful effect in all groups. What's more, it was the main cause of respiratory diseases among children and chronic diseases among people in all ages.

\section{EGMs visualisation}

The feature of EGMs is to provide a visual display of evidence from the SRs and MAs. A bubble plot was used to display the types of air pollution and related health outcomes in a two-dimensional matrix framework, as presented in figure 4 . The colours of the bubbles in the map represent the confidence of SRs and MAs, which were assessed by the SURE checklist. ${ }^{25}$ The red colour bubbles represent low confidence and the yellow colour bubbles show medium confidence. The bubble size indicates the relative number of included studies, a larger bubble represents a larger study sample size in each grid. The confidence result of identified studies is shown in online supplementary table S3. There was no green bubble in our EGM, which means that high-quality SRs and MAs were urgently needed. What's more, there was a correlation between the study sample size and research quality.

\section{DISCUSSION}

This EGM provides the most up-to-date SRs and MAs using user-friendly visualisation of the existing evidence in a matrix format of the interventions and outcomes framework; this helps to set potential research agendas and to promote evidence-based policymaking. EGM is a novel method for synthesis of evidence that offers a reliable means of covering a broad scope of a particular sector, focusing on visualising the quality of the existing evidence in a user-friendly format. Most importantly, EGM can address evidence gaps for funding research with limited resources and provide evidence-based information to support precise policymaking.

Most studies reported the health outcomes of cardiovascular diseases, respiratory diseases and health service records, which were mainly affected by fine particulate matter or combinations of multiple pollutants, while few studies investigated other toxic substances and their adverse effects on human health-related outcomes. By identifying the evidence gaps that existed in outdoor air pollution related health outcomes, more potential research agendas need to focus on chronic diseases, cancer and mental disorders, so that research gaps can be filled and evidence-based information can be provided by MA. By highlighting the confidence of the existing evidence based on inclusive studies, policymakers can promote relevant policies based on the existing summarised evidence of outdoor air pollution related health outcomes. In addition, more high-quality SRs and MAs, primary studies and synthesised evidence across a 
Table 5 Characteristics of included SRs and MAs based on health outcome groups

\begin{tabular}{|c|c|c|c|c|c|}
\hline $\begin{array}{l}\text { Health outcome } \\
\text { group } \\
\text { (number of SRs and } \\
\text { MAs) }\end{array}$ & $\begin{array}{l}\text { Types of air pollution* } \\
\text { (number of SRs and MAs) }\end{array}$ & $\begin{array}{l}\text { Number of } \\
\text { primary studies } \\
\text { (mean } \pm \text { STD) }\end{array}$ & $\begin{array}{l}\text { Population } \\
\text { group } \\
\text { (number of SRs } \\
\text { and MAs) } \\
\end{array}$ & $\begin{array}{l}\text { Sample size } \\
\text { (range) }\end{array}$ & Key results \\
\hline $\begin{array}{l}\text { Cardiovascular } \\
\text { diseases (24) }\end{array}$ & $\begin{array}{l}\text { Fine particulate matter (10) } \\
\text { General air pollution gas (1) } \\
\text { Combinations of multiple } \\
\text { pollutants (13) }\end{array}$ & $32 \pm 30$ & $\begin{array}{l}\text { Infants and } \\
\text { children (1) } \\
\text { Adults and old (5) } \\
\text { All ages (16) } \\
\text { Other (2) }\end{array}$ & $(83,11,850,884)$ & $\begin{array}{l}\text { Positive (22) } \\
\text { Ambiguous } \\
\text { (2) }\end{array}$ \\
\hline Chronic diseases (2) & $\begin{array}{l}\text { Combinations of multiple } \\
\text { pollutants (2) }\end{array}$ & $11 \pm-2$ & All ages (2) & $(402,62,012)$ & Positive (2) \\
\hline $\begin{array}{l}\text { Health service } \\
\text { records (22) }\end{array}$ & $\begin{array}{l}\text { Fine particulate matter (10) } \\
\text { General air pollution gas (4) } \\
\text { Other toxic substances (1) } \\
\text { Mixed air pollution ( } 7 \text { ) }\end{array}$ & $40 \pm 38$ & $\begin{array}{l}\text { Infants and } \\
\text { children (1) } \\
\text { Adults and old (1) } \\
\text { All ages (15) } \\
\text { Other (5) }\end{array}$ & $(1,050,50,756,699$ & $\begin{array}{l}\text { Positive (21) } \\
\text { Negative (1) }\end{array}$ \\
\hline Mental disorders (5) & $\begin{array}{l}\text { General air pollution gas (1) } \\
\text { Other toxic substances (1) } \\
\text { Mixed air pollution (3) }\end{array}$ & $16 \pm 10$ & $\begin{array}{l}\text { Infants and } \\
\text { children (2) } \\
\text { All ages (2) } \\
\text { Other (1) }\end{array}$ & $(252,7,203)$ & $\begin{array}{l}\text { Positive (3) } \\
\text { Negative (1) } \\
\text { Ambiguous } \\
\text { (1) }\end{array}$ \\
\hline $\begin{array}{l}\text { Respiratory diseases } \\
\text { (19) }\end{array}$ & $\begin{array}{l}\text { Fine particulate matter (3) } \\
\text { Other toxic substances (1) } \\
\text { Combinations of multiple } \\
\text { pollutants (15) }\end{array}$ & $37 \pm 31$ & $\begin{array}{l}\text { Infants and } \\
\text { children (11) } \\
\text { Adults and old (1) } \\
\text { All ages (6) } \\
\text { Other (1) }\end{array}$ & $(186,1,146,215)$ & $\begin{array}{l}\text { Positive (18) } \\
\text { Negative (1) }\end{array}$ \\
\hline
\end{tabular}

Combinations of multiple pollutants are the mixture of two or more of the following air pollutants: $\mathrm{O}_{3}, \mathrm{SO}_{2}, \mathrm{CO}, \mathrm{NO}_{2}, \mathrm{PM}_{2.5}, \mathrm{PM}_{10}$, suspended particulate matter and total suspended particles.

MAs, meta-analyses; SRs, systematic reviews.

range of disciplines are needed to ensure the confidence of existing evidence for making policy decisions.

This EGM is to comprehensively collect and categorise various SRs and MAs to overview the health outcomes affected by outdoor air pollution. However, the quality of the 86 included SRs and MAs is either medium or low. The visualised categories of outdoor air pollution and health outcomes reveal the research gaps and concentrations of the existing literature. The health outcomes that are mostly studied in outdoor air pollution research often concentrate on cardiovascular diseases, respiratory diseases and health service records, including chronic obstructive pulmonary disease (COPD), blood pressure, hypertension, asthma, morbidity and mortality. Most researchers and even the public are concerned about these diseases. The major air pollution types are fine particulate matter $\left(\mathrm{PM}_{25}\right.$ and $\left.\mathrm{PM}_{10}\right)$ and general air pollution gases $\left(\mathrm{CO}, \mathrm{SO}_{2}, \mathrm{NO}_{2}\right.$ and $\left.\mathrm{O}_{3}\right)$; few health outcomes are due to other toxic air pollutants. These measurements of air pollutants, as air quality standards developed by the US government in the 1970 s, are sometimes performed by different methods in other countries. This will induce some biases. There is a need for well-designed primary studies and MAs on chronic diseases, cancer and especially mental disorders. People with chronic diseases, such as diabetes or COPD, are more susceptible to air pollution. These chronic diseases are also age-related. The health outcomes of these patients affected by air pollution are worthy of further investigation.

Some research gaps have been explored in this EGM. A major gap observed is the lack of definite diagnostic criteria of diseases. Criteria and reporting of air pollution 


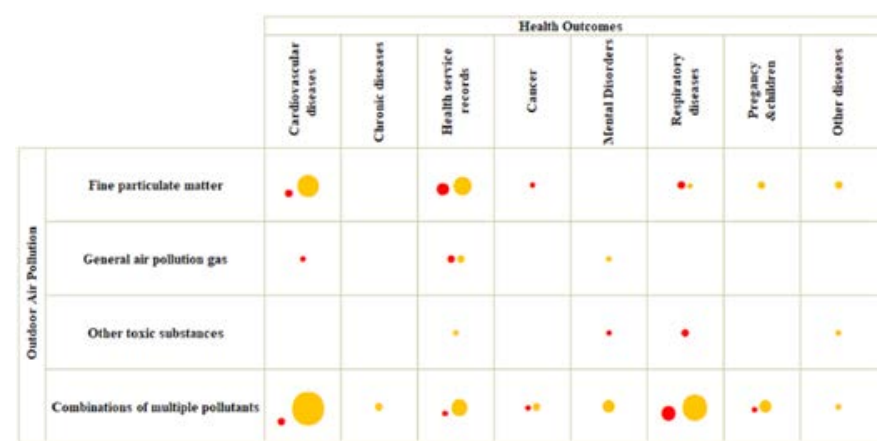

Figure 4 EGM for health outcomes affected by outdoor air pollution. The colours of the bubbles represent the confidence of included SRs and MAs; red colour represents low confidence and yellow colour represents medium confidence. The size of the bubbles indicates the relative number of included SRs and MAs, and a larger bubble represents a larger study sample size in each grid. EGM, evidence gap map; SRs, systematic reviews; MAs, metaanalyses.

related health outcomes are notably variable, with significant potential for subject heterogeneity. Future research should define and report the diagnostic criteria more precisely. Moreover, there are difficulties in estimating the personal exposure to air pollution. The heterogeneity of air pollution exposure levels, present in both the primary studies and MAs, may probably lead to the 'exposure bias'. Although more and more primary studies began to use the measurement of exposure time, there is still room to use more precise estimation, such as wearable sensor applications. Another research gap we found is that the studies' period of SRs was not clearly reported. The air pollution levels are different in different time periods. Even in the same country or region, the levels of industrialisation and modernisation are different, which makes air pollution effects different. These SRs and MAs should be updated, and the time period of SRs and MAs should also be clearly reported. A 'population bias' gap was also found in our EGMs. When including more children, for example, it could have overestimated the true prevalence of the diseases. A clearly stratified analysis of different age groups is needed because outdoor air pollution has different impacts on different age groups.

Some important areas of air pollution research are left blank in the EGM. For example, few of the SRs included healthcare costs related to outdoor air pollution. As public focus on environmental sustainability has become even stronger in the last few years, the demand for studies may increase. Moreover, they should come as a warning to policymakers as they show actual evidence for the healthcare costs related to outdoor air pollution. More precisely, studies focused on the economic burden of outdoor air pollution are needed.

Most of the included SRs and MAs estimated the health outcomes due to outdoor air pollution in the general population rather than in those with established disease, thus, more research summarising the existing body of evidence should be viewed, that aims at evaluating the health outcomes of established diseases affected by outdoor air pollution. Besides, research should be focused on the physiological effects, such as increased heart rates and feelings of anxiety, which are indispensable, so that EGM can capture a comprehensive overview of air pollution related health outcomes. Geographically, it is worth noting that most of the available primary studies included in SRs and MAs were conducted in western countries, where the median $\mathrm{PM}_{2.5}$ concentration is less than $20 \mu \mathrm{g} /$ $\mathrm{m}^{3}$. In developing countries (such as China and India), however, the $\mathrm{PM}_{2.5}$ concentrations in urban cities are likely to be up to $100 \mu \mathrm{g} / \mathrm{m}^{3}$, which has greatly impacted the local environment and people's health outcomes. The location of the primary study is an impact factor related to air pollution, and it should be considered with caution in both epidemiological studies and SRs. There is room for robust impact evaluation in the process of SRs by using subgroup analysis.

There have been some gaps in the current research on the effects of air pollution on people's health outcomes. Uniform diagnostic criteria of diseases, timely updating well-designed primary studies in local countries and rational sampling (elders, children and patients with chronic diseases) should be considered with caution during the evidence-based policy decision making process. These factors will affect the research outcomes by inducing some unobserved bias.

\section{Limitations}

Our EGM was built on the evidence from significant numbers of SRs and MAs that have quantitatively addressed the health outcomes influenced by air pollution. The 'gold standard' restriction on included studies may neglect important information available from studies. Since the main purpose of our EGMs is to provide a resource for policymakers, only the inclusion of SRs and MAs is sufficient to generate reliable conclusions. ${ }^{20}$ Hundreds of quality appraisal methods exist in research synthesis studies; ${ }^{26}$ the efficiency of these studies depends largely on the quality assessment results. We only applied the SURE checklist instead of other quality assessment methods because it is more suitable for EGMs. Two reviewers performed the assessments separately to prevent any bias.

Only English-language SRs and MAs were included in our EGM, thus possibly missing pertinent information written in other languages. Primary studies, such as observational studies, time-series studies and causal inference studies were not included since systematic evidence has been comprehensively collected from the current research. In addition, this EGM largely relied on information provided by included SRs and MAs. There may have been some undetected errors in data extraction or research synthesis. Finally, research gaps identified in this EGM do not necessarily equate to research needs. The desirability, feasibility and importance of the research gaps should be considered, highlighting the importance of stakeholder engagement in this process. 


\section{CONCLUSIONS}

This EGM provides a visual overview of health outcomes affected by outdoor air pollution. Despite the outlined limitations, it is a useful tool to inform environmental policy decision-makers. It also can promote further potential research by visualising and synthesising high-quality SRs and highlighting the absolute gaps. More prospective studies, with large numbers of participants, are needed in developing countries. It will be helpful to assess the longterm effects of air pollution more precisely.

Contributors ZS formulated the research question. DZ and ZS devised the search strategy, consulting with experts from the Committee on Public Health and Urban Environment Management in China. DZ and ZS conducted the search, screened all titles, abstracts and full articles and extracted data. DZ and ZS co-wrote the manuscript.

Funding This work was supported by a major project of the National Social Science Fund of China grant number 13\&ZD176.

Competing interests None declared.

Patient consent for publication Not required.

Provenance and peer review Not commissioned; externally peer reviewed.

Data availability statement All data relevant to the study are included in the article or uploaded as supplementary information.

Open access This is an open access article distributed in accordance with the Creative Commons Attribution Non Commercial (CC BY-NC 4.0) license, which permits others to distribute, remix, adapt, build upon this work non-commercially, and license their derivative works on different terms, provided the original work is properly cited, appropriate credit is given, any changes made indicated, and the use is non-commercial. See: http://creativecommons.org/licenses/by-nc/4.0/.

ORCID iD

Zhuanlan Sun http://orcid.org/0000-0003-4958-7351

\section{REFERENCES}

1 WHO (World Health Organization). Air pollution, 2016. Available: http://www.who.int/airpollution/en/

2 Berend N. Contribution of air pollution to COPD and small airway dysfunction. Respirology 2016;21:237-44.

3 Katsoulis M, Dimakopoulou K, Pedeli X, et al. Long-term exposure to traffic-related air pollution and cardiovascular health in a Greek cohort study. Sci Total Environ 2014;490:934-40.

4 Jung KH, Torrone D, Lovinsky-Desir S, et al. Short-term exposure to $\mathrm{PM}_{2.5}$ and vanadium and changes in asthma gene DNA methylation and lung function decrements among urban children. Respir Res $2017 ; 18$.

5 Downward GS, Hu W, Rothman N, et al. Quartz in ash, and air in a high lung cancer incidence area in China. Environ Pollut 2017;221:318-25.
6 Calderón-Garcidueñas L, Reed W, Maronpot RR, et al. Brain inflammation and Alzheimer's-like pathology in individuals exposed to severe air pollution. Toxicol Pathol 2004;32:650-8.

7 Peters MDJ. In no uncertain terms: the importance of a defined objective in scoping reviews. JBI Database System Rev Implement Rep 2016;14:1-4.

8 Takenoue Y, Kaneko T, Miyamae T, et al. Influence of outdoor $\mathrm{NO}_{2}$ exposure on asthma in childhood: meta-analysis. Pediatr Int 2012;54:762-9.

9 Zheng X-yan, Ding H, Jiang L-na, et al. Association between air pollutants and asthma emergency room visits and hospital admissions in time series studies: a systematic review and metaanalysis. PLoS One 2015;10:e0138146.

10 Chen H, Goldberg MS, Villeneuve PJ. A systematic review of the relation between long-term exposure to ambient air pollution and chronic diseases. Rev Environ Health 2008;23:243-97.

$11 \mathrm{Lin} \mathrm{H}$, Liu T, Xiao J, et al. Mortality burden of ambient fine particulate air pollution in six Chinese cities: results from the pearl River delta study. Environ Int 2016;96:91-7.

12 Zhang S, Li G, Tian L, et al. Short-term exposure to air pollution and morbidity of COPD and asthma in East Asian area: a systematic review and meta-analysis. Environ Res 2016;148:15-23.

13 Weichenthal S, Bai L, Hatzopoulou M, et al. Long-term exposure to ambient ultrafine particles and respiratory disease incidence in in Toronto, Canada: a cohort study. Environmental Health 2017;16.

14 Mölter A, Simpson A, Berdel D, et al. A multicentre study of air pollution exposure and childhood asthma prevalence: the escape project. Eur Respir J 2015;45:610-24.

15 Dick S, Doust E, Cowie H, et al. Associations between environmental exposures and asthma control and exacerbations in young children: a systematic review. BMJ Open 2014;4:e003827.

16 Arksey H, O'Malley L, 'Malley L O. Scoping studies: towards a methodological framework. Int J Soc Res Methodol 2005;8:19-32.

17 James KL, Randall NP, Haddaway NR. A methodology for systematic mapping in environmental sciences. Environ Evid 2016;5:7.

18 Bragge P, Clavisi O, Turner T, et al. The global evidence mapping initiative: Scoping research in broad topic areas. BMC Med Res Methodol 2011;11:92.

19 McKinnon MC, Cheng SH, Garside R, et al. Sustainability: map the evidence. Nature 2015;528:185-7.

20 Snilstveit B, Vojtkova M, Bhavsar A, et al. Evidence gap maps a tool for promoting evidence-informed policy and prioritizing future research 2013.

21 Snilstveit B, Vojtkova M, Bhavsar A, et al. Evidence \& Gap Maps: A tool for promoting evidence informed policy and strategic research agendas. J Clin Epidemiol 2016;79:120-9.

22 Schmucker C, Motschall E, Antes G, et al. [Methods of evidence mapping. A systematic review]. Bundesgesundheitsblatt Gesundheitsforschung Gesundheitsschutz 2013;56:1390-7.

23 Grimshaw JM, Eccles MP, Lavis JN, et al. Knowledge translation of research findings. 7, 2012.

24 Bilotta GS, Milner AM, Boyd IL. Quality assessment tools for evidence from environmental science. Environ Evid 2014;3:14.

25 The SURE Collaboration. Sure checklist for making judgments about how much confidence to place in a systematic review. in: sure guides for preparing and using policy briefs. version 2.1. Available: www. evipnet.org/sure [Accessed 28 Sep 2016].

26 Wells K, Littell JH. Study quality assessment in systematic reviews of research on intervention effects. Res Soc Work Pract 2009;19:52-62. 